\title{
Combined analysis of gene expression and genome binding profiles identified potential therapeutic targets of ciclopirox in Ewing sarcoma
}

\author{
BAISHENG YUAN, WEI JI, HAIPENG XIA and JIANMIN LI
}

Department of Orthopaedics, Qilu Hospital of Shandong University (Qingdao), Qingdao, Shandong 266035, P.R. China

Received June 2, 2017; Accepted December 11, 2017

DOI: $10.3892 / \mathrm{mmr} .2018 .8418$

\begin{abstract}
Ciclopirox (CPX) is a synthetic antifungal drug that is mainly used to treat dermatomycoses. The aim of the present study was to determine whether CPX could influence Ewing sarcoma progression. The present study suggested that CPX treatment may inhibit Ewing sarcoma (ES) progression through Ewing sarcoma breakpoint region 1-Friend leukemia integration 1 (EWS-FLI1), a common fusion transcript structure in patients with ES. To determine the underlying mechanisms of ES progression, cross analysis was conducted on three high-throughput genome or transcript me datasets from the Gene Expression Omnibus. The results indicated that CPX may inhibit ES growth by affecting vasculature development and DNA replication. A combination of genome-wide expression and binding profiles revealed several potential targets for CPX in ES, including collagen type I $\alpha 2$ chain, $\mathrm{N}$-myc proto-oncogene and transforming growth factor $\beta 1$, which contained significantly enriched binding peaks of FLI1. In addition, network analysis, including a protein-protein interaction network and a transcription regulatory network, provided further detailed information about the roles of CPX in ES. This study may provide a novel solution for ES treatment and may also aid in improving its prognosis.
\end{abstract}

\section{Introduction}

Ewing sarcoma (ES) is the second most common malignant bone tumor, with the highest morbidity rates in children aged 10-20 years and with undistinguishable features from another Ewing family tumor, primitive neuroectodermal tumor (PNET) $(1,2)$. Surgery, radiation and chemotherapy make up

Correspondence to: Professor Jianmin Li, Department of Orthopaedics, Qilu Hospital of Shandong University (Qingdao), 758 Hefei Road, Qingdao, Shandong 266035, P.R. China

E-mail: gkljm@163.com

Key words: chromatin immune precipitation followed by high-throughput sequencing, ciclopirox, Ewing sarcoma, Ewing sarcoma breakpoint region 1-Friend leukemia integration 1 fusion transcript, Gene Expression Omnibus the standard treatments of ES; although there has been significant improvement in prognosis for localized ES, the survival rates of metastatic patients are low with only $13-30 \%$ 5-year survival rate $(3,4)$. Early diagnosis would aid in improving the prognosis of patients with ES, whereas traditional pathological diagnosis has a number of limitations, such as subjectivity and an absence of biological markers (5). Besides, there are 200 new cases among children and 20 new cases among adults in USA per year. Therefore, it is necessary to develop novel diagnostic and treatment methods to reduce ES morbidity and mortality.

The development of genomic technologies has provided unprecedented opportunities to detail the progression of ES and biomarker identification. A common biomarker target in ES is Ewing sarcoma breakpoint region 1-Friend leukemia integration 1 (EWS-FLI1), a fusion gene induced by $\mathrm{t}(11 ; 22)$ (q24;q12) chromosome translocation (6-8), which has been reported as an independent prognosis determinant in ES (9). EWS-FLI1 may directly activate or repress multi gene enhancer elements associated with ES through different chromatin remodeling mechanisms that may promote tumor cell growth and proliferation (10). In addition, as a transcription factor, EWS-FLI1 binds to various genes and determines drug response (11); and based on this information several small bioactive molecules were developed for the treatment of ES (12-14). Ciclopirox (CPX) is an antifungal drug that isoften used for the treatment of fungal-induced skin and nail diseases (15-17). A previous study reported on the antitumoral activity of CPX in human breast cancer through a series molecular level analyses, which may indicate its potential use in cancer treatment (18). Another study demonstrated the inhibitory activity of CPX on mammalian target of rapamycin, which is useful for promoting parthenolide anti leukemia activity (19). However, there are few studies concerning the roles of CPX in ES.

In the present study, the roles of CPX in ES were explored through the combined analysis of genome and transcript me datasets, and for the first time, to the best of our knowledge, combined analysis of a CPX-regulated gene expression profile and an EWS-FLI1 genome-binding profile was conducted. The transcript me datasets comprised oxicycl in induced EWS-FLI1-knockdown and wild-type ES samples (A673 cell lines without any treatment), which aided in identifying targets that may be regulated by EWS-FLI1. The genome 
datasets are chromatin immune precipitation coupled with high-throughput sequencing (ChIP-seq) data of EWS-FLI1 in CPX-treated A673 cell line. Through the genome datasets, we should identify CPX-specific targets mediated by EWS-FLI1. Therefore, the combined analysis of the two datasets may be able to obtain more reliable targets of CPX in ES. In addition, the systemic bioinformatics analysis performed in the present study, which included functional and network analysis, may aid our understanding of the underlying mechanisms of ES and to improve prognosis.

\section{Materials and methods}

High-throughput datasets. The present study used three high-throughput genome and transcript me datasets that were downloaded from the Gene Expression Omnibus online repository (https://www.ncbi.nlm.nih.gov/geo/). To investigate the influence of EWS-FLI1 knockdown on genome-scale expression, gene expression profiles in A673 ES cell lines with the accession number of GSE27524 (20), which contained six time points $(0,18,36,53,72$ and $96 \mathrm{~h})$ following EWS-FLI1 doxycycline-inducible knockdown, and a total of 22,277 transcripts were profiled based on the Affymetrix Human Genome U133A 2.0 Array. Another genome-scale expression dataset, GSE79641 (21), was used to explore the influence of CPX treatment in ES; this data set comprised 36 ES cell lines, including 3CPX-treated A673 cell lines and three corresponding dimethylsulfoxide-treated A673 cell lines as controls. In addition, two sub-data sets of GSE61944 (10), including GSM1517562, which was obtained through EWS-FLI1 chromatin immune precipitation followed by high-throughput sequencing (ChIP-seq) in A673, and GSM1517568, which was from A673 whole-cell extracts, were used for the identification of EWS-FLI1 specific binding sites (peaks).

Gene expression profiles analysis. Raw gene expression profiles were normalized according to the method described by Yang et al (22). Probesets were translated to gene symbol through the gene microarray annotation file, and the expression values for genes corresponding to multi-probesets were summarized. The differentially expressed genes (DEGs) in EWS-FLI1-knockdown and CPX-treated A673 cell lines were identified using the linear models for microarray and RNA-sequencing data (limma; http://www.bioconducto r.org/packages/release/bioc/html/limma.html), an open source $\mathrm{R}$ programming package using t-test for expression values in EWS-FLI1 knockdown and control A673 cell lines, and using the criteria of fold change $>2$ or $<0.5$, i.e. $\mid \log 2 \mathrm{FCl}>1$ and false discovery rate $<0.05$. Based on Heatplusbio conductor package (https://www.bioconductor.org/packa ges/release/bioc/html/Heatplus.html), we conducted two-way hierarchical clustering analysis of DEGs and their expression profiles in all of the samples.

Functional enrichment analysis. To investigate the biological processes that may be influenced by EWS-FLI1 or CPX in ES, gene ontology (GO) term functional enrichment analysis was conducted for the DEGs identified from EWS-FLI1-knockdown and CPX-treated A673 cell lines through the Web Gestalt (23) online database. In this study, only the biological processes that satisfied the criteria of $\mathrm{P}<0.05$ and hits count $>5$ were obtained. For the interpretation of significantly enriched GO terms, we submitted them to REVIGO online tool (24) with the default thresholds, i.e. medium similarity (0.7) between GO terms.

Identification of EWS-FLII specific binding sites. The present study identified EWS-FLI1-specific binding sites, which were referred as peaks, through the analysis of EWS-FLI1 ChIP-seq datasets. First, quality control was performed for the raw datasets using Fast QC (http://www. bioinformatics.babraham.ac.uk/projects/fastqc), and only those reads that contained $>90 \%$ bases with a satisfactory quality score $>20$ were retained. Second, the remaining reads were mapped to the hg19 reference genome obtained from the UCSC Genome Browser (http://genome.ucsc.edu/) using Bowtie version 2 with maximum mismatches of 2 (25). Third, significantly enriched peaks were identified with the Model-based Analysis of ChIP-seq (MACS; version2) (26), following the removal of duplicated mappings with the default thresholds, including Q-value (FDR adjusted P-value) $<0.01$. Finally, the peaks were annotated with their corresponding genomic features using the ChIP seeker package in $\mathrm{R}$, in which the region spanning 3,000 bp downstream and upstream of the transcription start site was considered to be the promoter region (27). Binding profiles of EWS-FLI1 in certain target genes were further visualized using Integrative Genomics Viewer (IGV) (28).

Network analysis. Genes shared between DEGs of EWS-FLI1-knockdown and CPX-treated A673 cell lines, which were referred as DECs, were considered as common targets of EWS-FLI1 and CPX in ES. To obtain more reliable results, DECs and genes that were specifically bound by EWS-FLI1 in their promoters were intersected, and the overlaps were considered as reliable EWS-FLI1 targets (EFTs) in A673 cells. The potential interaction pairs among EFTs were obtained through the STRING database (29) with the minimum combined score $>0.4$. In addition, the predicted regulatory relationships between EWS-FLI1 and the EFTs, and interaction pairs among the EFTs were visualized with Cy to scape software (30).

\section{Results}

Differential expression analysis. For the GSE27524EWS-FLI1-knockdown A673 cell lines, 5 lists of DEGs were identified for the 5 time points $(18,36,53,72$ and $96 \mathrm{~h}$ ) when they were compared with the $0 \mathrm{~h}$ cells following EWS-FLI1 knockdown, and these were referred as DEG18, DEG36, DEG53, DEG72 and DEG96, respectively. Volcano plots of the corresponding fold change ( $\log 2$ scale) against the transformed (-log10) Q-values for every time point are illustrated in Fig. 1A-E. All volcano plots complied with normal distribution with fold-change values ranging between-6.4 and 12.0 for both downregulated and upregulated genes. The volcano plot changes as the length of time increases post-knockdown. The $18 \mathrm{~h}$ plot appears similar to the $36 \mathrm{~h}$ plot, whereas the later time points of 53,72 and $96 \mathrm{~h}$ are more similar to each other, particularly in the upregulated expression area 
A

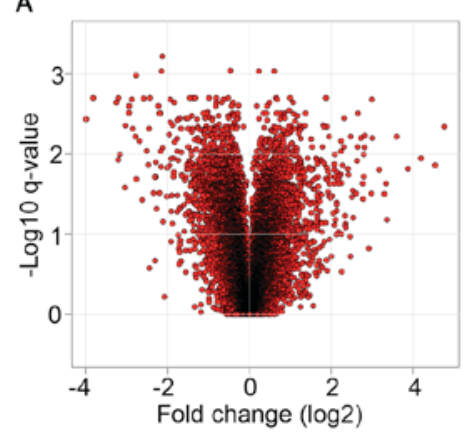

C

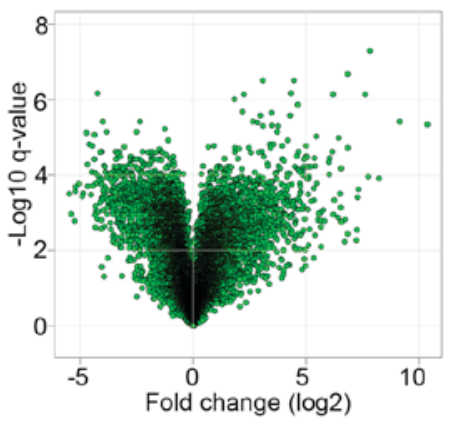

E

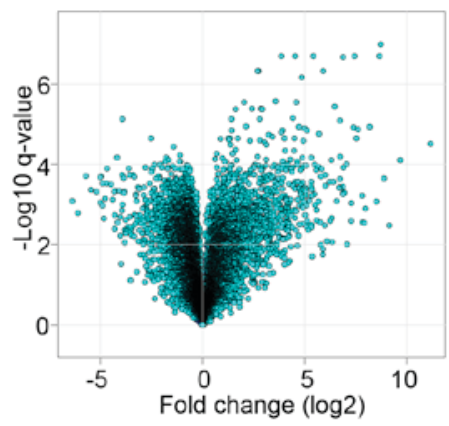

B

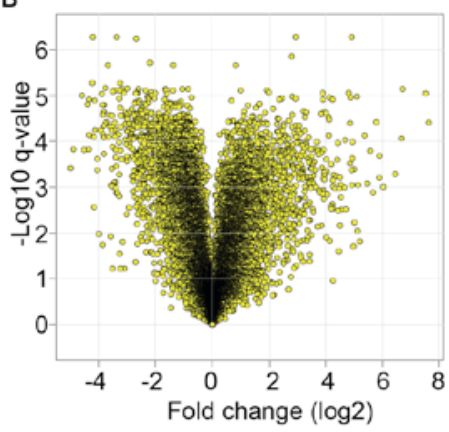

D
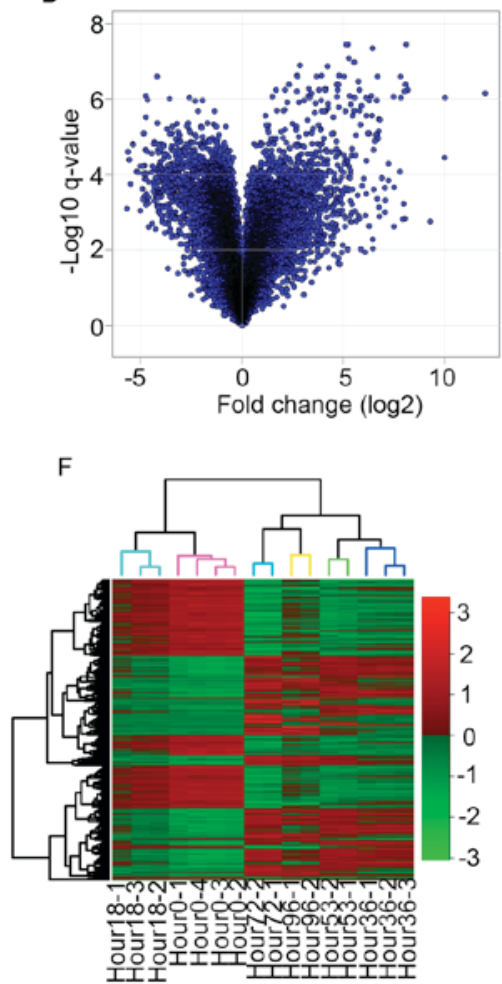

Figure 1. Time point gene expression profile analyses. (A-E) Volcano plots of fold change (log2) values of all genes vs. transformed (-log10) t-test Q-values. Similar patterns are observed between (A) the $18 \mathrm{~h}$ and (B) the $36 \mathrm{~h}$ time points, and between the (C) $53 \mathrm{~h}$, (D) $72 \mathrm{~h}$ and (E) $96 \mathrm{~h}$ plots. (F) Unsupervised clustering of different time points by expression. The results were visualized with the Heat plus package of BioConductor, with columns indicating the samples and rows representing genes. Green and red color indicate decreased and increased expression value respectively. All samples are divided into two groups, 0 and $18 \mathrm{~h}$ form one cluster, the remaining time points form another cluster.

(right side of volcano plots). This observation was probably due to the knockdown of EWS-FLI1 resulting in upregulated expression of tumor suppressor genes. Unsupervised clustering based on the expression profiles of the five lists of DEGs obtained consistent pattern with volcano plots (Fig. 1F); two main clusters are obtained: 0 and $18 \mathrm{~h}, 36,53,72$ and $96 \mathrm{~h}$. The two main clusters were each further subdivided according to different time points.

The relationships among different groups of DEGs were also examined by creating Venn diagrams with overlapping regions that represent the overlapping DEGs (Fig. 2). The gene contents were more similar among the later time points, in which 973 overlapped genes were identified among the 36, 72 and 96 h DEGs. For CPX-treated A673 cell lines, a total of 466 DEGs (referred as DEG-CPX) were obtained, which comprised 167 downregulated genes and 299 upregulated genes.
Enriched functions. The 5 lists of DEGs that were identified in the EWS-FLI1-knockdown A673 cell lines were combined and the duplicates were removed, which resulted in 4,500 unique DEGs. A total of 635 biological process (BP) GO terms with minimum hits $>5$ were indicated to be significantly enriched in those genes. The 466 identified DEG-CPX DEGs were closely associated with 182 BP GO terms. The GO terms present level information based on GO database (http://amigol.geneontology.org/cgi-bin/amigo/go.cgi) where the root terms, biological process, molecular function and cellular component, have level 1 and terms with higher level could indicate more specific function. It is helpful to remove redundant information for the interpretation of enriched functions. For this purpose, the full lists of BP GO terms were submitted to REVIGO online tool to refine the enriched functions of DEGs. As a result, the 4,500 EWS-FLI1-knockdown DEGs are revealed to be closely associated with BPs that 
A

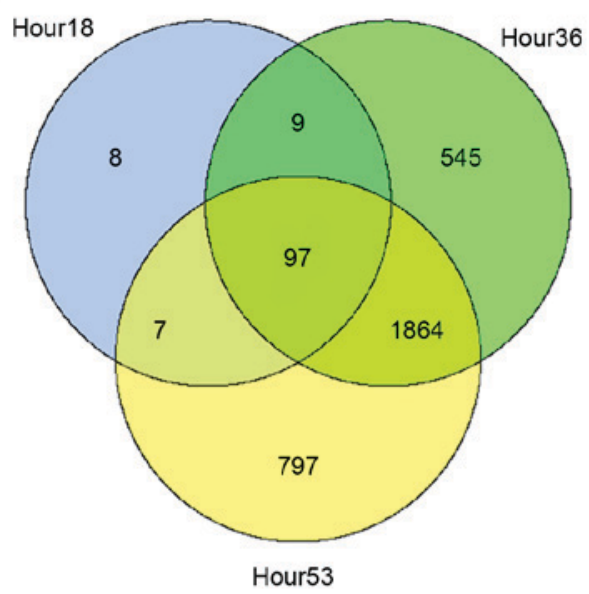

C

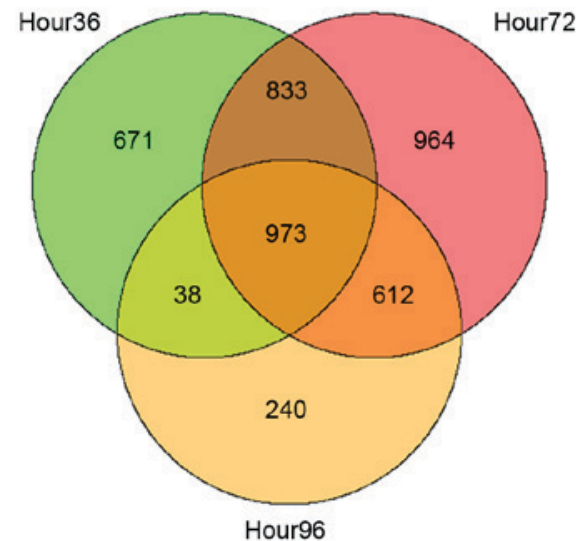

B

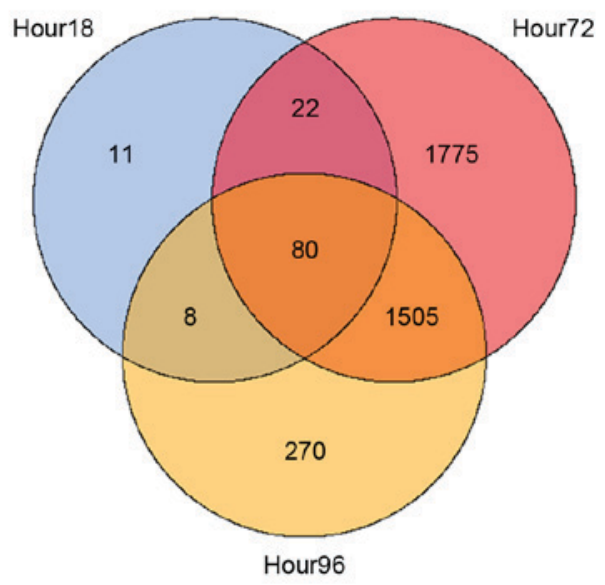

Figure 2. Venn diagrams of the intersection of DEGs of different time points. (A-C) Relationship between the DEGs at different time points with the thresholds of fold change $(\log 2$ scale $)>2$ and $\mathrm{P}<0.05$. DEG, differentially expressed gene.

are related to cell activity, chromosome structure and DNA replication (Fig. 3A). The 466 DEG-CPXDEGs are closely associated with vasculature development, cell activity and response to drug (Fig. 3B).

EWS-FLII specific binding sites. The present study used MACS version 2 to determine the binding profiles of EWS-FLI1 in ES cell lines, in which binomial distribution is used to testing enrichment significance of the binding sites. ChIP-seq analysis identified 3,885 peaks for EWS-FLI1, most of which mapped to the distal intergenic region (Fig. 4A); however, the strongest binding signals were detected in the promoter region (Fig. 4B), which indicated more reliable targets of EWS-FLI1. DECs were intersected with genes that contained EWS-FLI1 binding peaks in their promoters and 34 overlaps, that is EFTs. Table I shows the CPX-induced fold change (log2 scale) of the 34 EFTs, of which 8 of them were revealed to be downregulated by CPX treatment. To illustrate the binding profiles of EWS-FLI1 on the 34 EFTs, 3 targets were randomly selected, collagen type I $\alpha 2$ chain (COL1A2), N-myc proto-oncogene and transforming growth factor $\beta 1$ (TGF $\beta 1$ ), and the EWS-FLI1 binding signals on them were visualized using the Integrative Genomics Viewer (IGV). The results demonstrated that certain parts of these three targets exhibited strong binding signals in their promoter regions (Fig. 4C). For MYCN, a strong binding signal was obtained across the whole gene body (Fig. 4C).

Network analysis. The 34 EFTs were considered as potential therapeutic targets of CPX in ES and, as such, it was hypothesized that they may interact with each other to promote or suppress the progression of ES. A total of 10 interaction pairs were identified among the 34 EFTs, and a combined network of the 10 interaction pairs and the regulatory relationships between EWS-FLI1 and the 34 EFTs was created (Fig. 5).

\section{Discussion}

In 1973, Dittmar and Lohaus (31) first reported the antifungal functions of CPX, and it has since been widely used to treat fungal-induced skin and nail diseases $(16,32,33)$. In addition, a number of subsequent studies have also demonstrated the antitumoral activity of CPX, particularly in hematologic malignancies $(17,34)$; however, only a few studies were focused the activity of CPX on solid tumors $(35,36)$. The present study conducted combined analysis of gene expression and genome binding profiles of CPX and EWS-FLI1 to screen potential valuable therapeutic targets of CPX in ES. These results may 
Table I. Ciclopirox-induced FC ( $\log 2$ scale) of the 34 identified Ewing sarcoma breakpoint region 1-Friend leukemia integration 1 target genes.

\begin{tabular}{lc}
\hline Gene & FC (log2 scale) \\
\hline GYG2 & -1.74 \\
ZNF423 & -1.74 \\
COL1A2 & -1.69 \\
MN1 & -1.30 \\
TRAF3IP2 & -1.23 \\
MAP2K5 & -1.10 \\
SLITRK5 & -1.03 \\
LMO3 & -1.02 \\
JMJD1C & 1.02 \\
DKK2 & 1.06 \\
TWIST1 & 1.06 \\
TLE4 & 1.07 \\
CLDN1 & 1.08 \\
MYCN & 1.09 \\
LYPD1 & 1.14 \\
TGFBI & 1.21 \\
SPRY2 & 1.23 \\
CTH & 1.24 \\
LAMB3 & 1.25 \\
DNAJC12 & 1.26 \\
MMP10 & 1.28 \\
PLOD2 & 1.28 \\
FN1 & 1.29 \\
TMEM45A & 1.42 \\
KLF6 & 1.44 \\
TSC22D3 & 1.56 \\
SLC2A3 & 1.58 \\
COL11A1 & 1.78 \\
NDRG1 & 1.93 \\
RFTN1 & 2.01 \\
ARC & 2.14 \\
TNFAIP3 & 2.22 \\
KLF5 & 2.42 \\
STC1 & 2.50 \\
\hline
\end{tabular}

FC, fold change.

be helpful for the comprehensive understanding of the roles of CPX and EWS-FLI1 in ES development.

EWS-FLI1 is a fusion transcript structure caused by chromosome translocation of $\mathrm{t}(11 ; 22)$ (q24;q12), which was previously demonstrated to be closely associated with the initiation and progression of ES $(11,12,37,38)$. In the present study, the combination of gene expression profiles of CPX-treated and EWS-FLI1-knockdown ES A673 cell lines was used to identify genes that were influenced by CPX treatment and EWS-FLI1, that is, DECs. To make the results more reliable, the potential binding targets of EWS-FLI1 in A673 cell lines were identified through ChIP-seq analysis and these targets were intersected with the DECs; the resulting

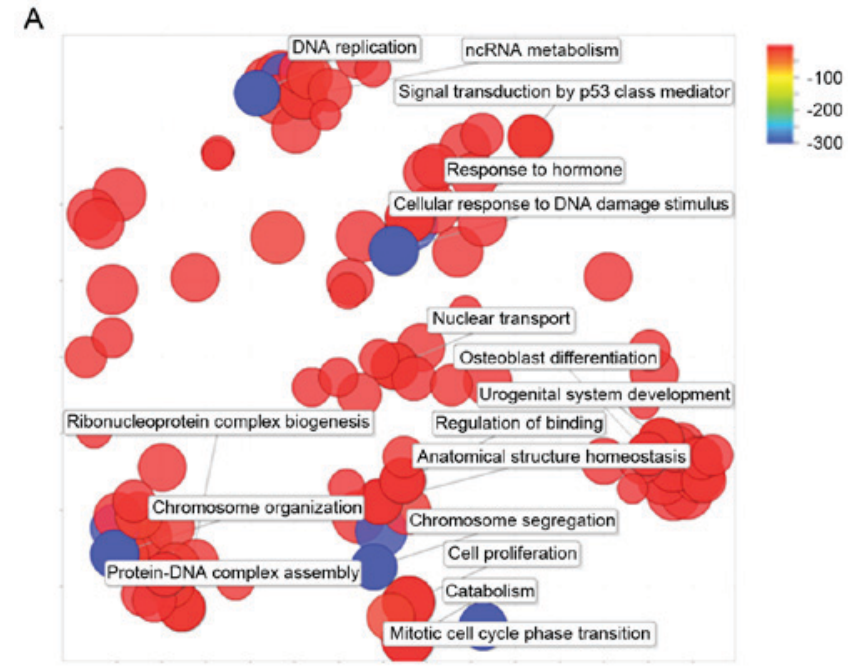

B

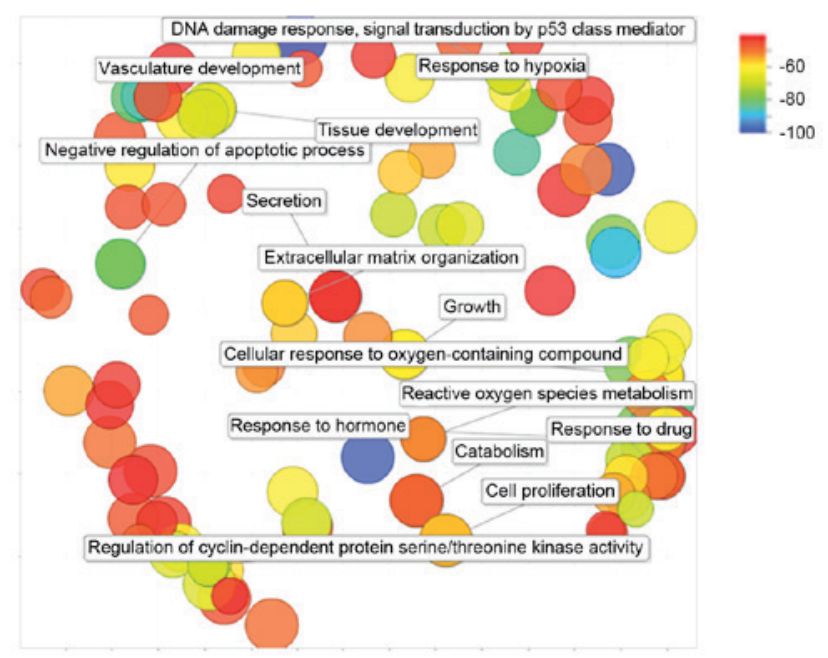

Figure 3. BPGO term functional enrichment analysis. (A) Significantly enriched BP GO terms of DEGs in EWS-FLI1-knockdown ES A673 cell lines. (B) Significantly enriched BP GO terms of DEGs in CPX-treated ES A673 cell lines. Different colors indicate the different enrichment significance with colors from blue to red representing decreasing significance. The full list GO terms were submitted to REVIGO online tool for the removal of duplicates and visualization. BP, biological process; CPX, ciclopirox; GO, gene ontology; EWS-FLI1, Ewing sarcoma breakpoint region 1-Friend leukemia integration 1; ES, Ewing sarcoma.

overlaps, the EFTs, should be CPX-targeted genes in ES through EWS-FLI1. Network analysis identified COL1A2, TWIST1 and TGF $\beta 1$ as core nodes for they have more direct neighborhoods. Consistent with the present study results, these three genes were previously demonstrated to be regulated by EWS-FLI1 and interacted with each other (39-42). TGF $\beta 1$ promotes expression of COL1A2 and is involved in the progression of several diseases, including lung fibrosis (43), skin fibrosis (44) and dermal fibrosis (45), and the inhibited expression of TGF $\beta$-type genes by EWS-FLI1 is an important cause of ES initiation (46). Treatment with CPX also resulted in the upregulation of TGF $\beta 1$, which indicated a putative role of TGF $\beta 1$ in ES.

DEGs that were influenced by EWS-FLI1-knockdown were closely associated with BPs related to cell activity and DNA replication, which may affect the progression of numerous forms of cancer. DEGs in the CPX-treated A673 
A

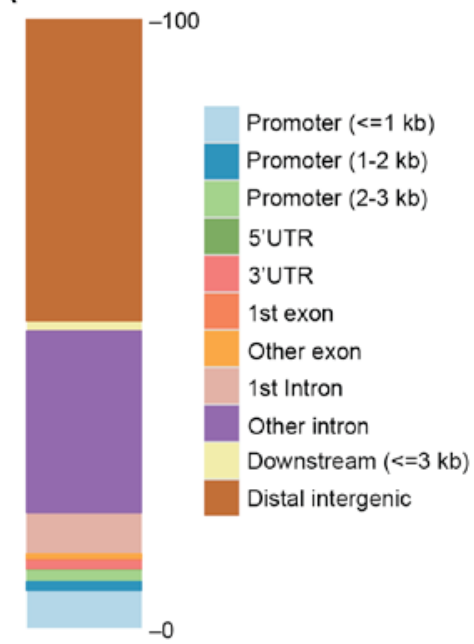

B

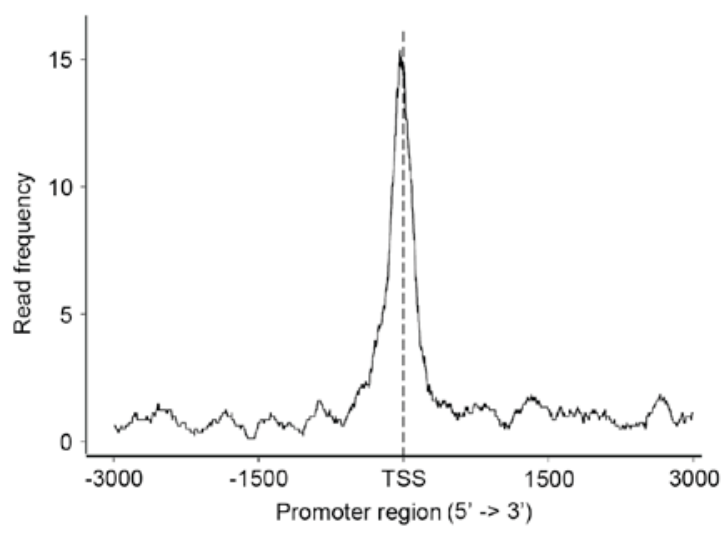

C

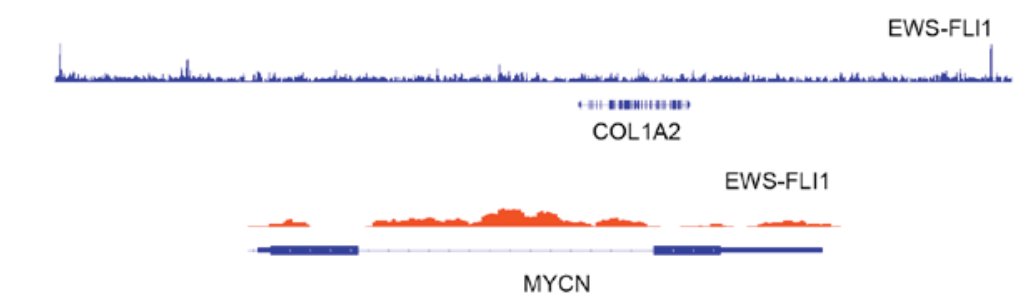

EWS-FLI1

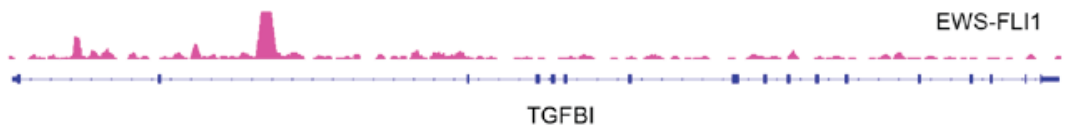

Figure 4. EWS-FLI1 ChIP-seq datasets analysis. (A) Genome distribution of EWS-FLI1 specific binding sites visualized by ChIP seeker. A majority of the peaks are located in the distal intergenic regions, and a small number of peaks are located in promoter regions. (B) Binding-density profiles of EWS-FLI1 across genome promoter regions. A significant peak exists in the TSS. (C) Binding profiles of EWS-FLI1 on COL1A2, MYCN and TGFBI visualized by Integrative Genomics Viewer. Significant binding profiles were identified in the promoter region or the whole gene body. COL1A2, collagen type I $\alpha 2$ chain; ChIP-seq, chromatin immunoprecipitation followed by high-throughput sequencing; EWS-FLI1, Ewing sarcoma breakpoint region 1-Friend leukemia integration 1; MYCN, N-mycproto-oncogene; TGF $\beta 1$, transforming growth factor $\beta 1$; TSS, transcription start site; UTR, untranslated region.

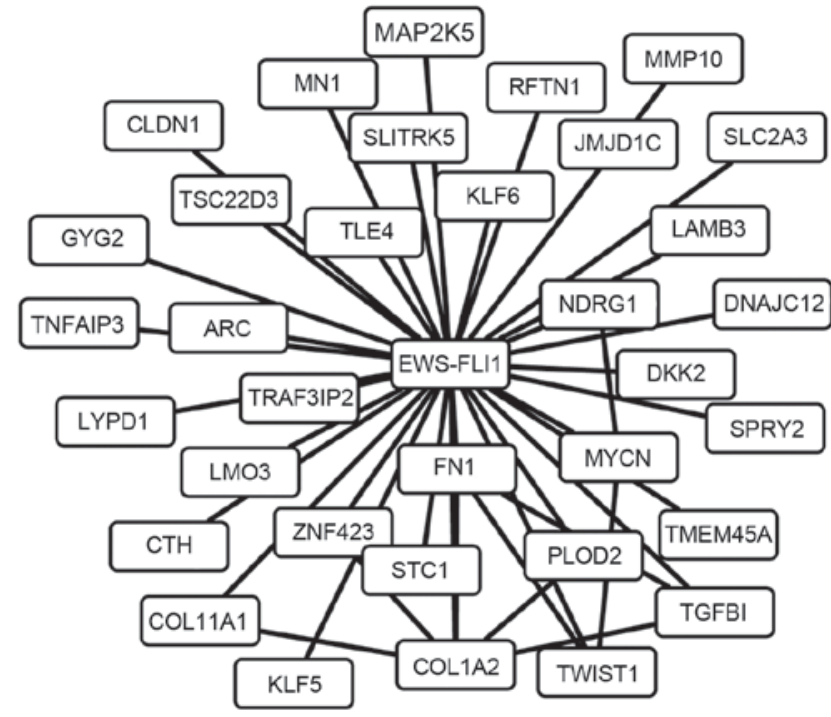

Figure 5. Network analysis by the combination of transcription regulation network (EWS-FLI1 and the 34 EWS-FLI1 targets) and protein-protein network of DEGs. EWS-FLI1, Ewing sarcoma breakpoint region 1-Friend leukemia integration 1; DEG, differentially expressed genes. cell lines were associated with tissue development, particularly vascular development. Vasculature is important for oxygen and nutrient transportation for cancer cells (47-49). TWIST1 is one of the core genes in the network and serves a role in embryonic vascular development and tumor metastasis, and it is a direct target of hypoxia-inducible factor- $2 \alpha$ (HIF2 $\alpha)(50,51)$. HIF2 $\alpha$ is closely associated with oxygen metabolism in cancer, which is associated with cancer progression $(52,53)$. In the present study, TWIST1 is a core gene of the network and it was previously reported to be a direct target of HIF $2 \alpha$, so it may be important for the progression of ES. Therefore, CPX may influence the progression of ES by controlling the process of vascular development. In this study, MYCN was identified to be a target of EWS-FLI1 with significantly enriched peaks across the gene body. MYCN is a member of the MYC family and encodes a protein with a basic helix-loop-helix (bHLH) domain. The amplification of MYCN is closely associated with a number of cancer types, including ES (54). The present study demonstrated that CPX could affect progression of ES by regulating the binding of EWS-FLI1 
in MYCN for the enrichment of EWS-FLI1 binding sites in MYCN in CPX-treated A673 cell line.

In conclusion, the present study performed systemic analysis of gene expression and genome binding profiles in ES A673 cell lines. The results may shed light on the influence of EWS-FLI1 on ES development and progression, and may provide a novel therapeutic method, such as CPX-target therapy, which may aid in improving the prognosis of patients with ES.

\section{References}

1. Grier HE: The Ewing family of tumors. Ewing's sarcoma and primitive neuroectodermal tumors. Pediatr Clin North Am 44: 991-1004, 1997.

2. Esiashvili N, Goodman M and Marcus RB Jr: Changes in incidence and survival of Ewing sarcoma patients over the past 3 decades: Surveillance epidemiology and end results data. J Pediatr Hematol Oncol 30: 425-430, 2008.

3. Rosen G, Wollner N, Tan C, Wu SJ, Hajdu SI, Cham W, D'Angio GJ and Murphy ML: Proceedings: Disease-free survival in children with Ewing's sarcoma treated with radiation therapy and adjuvant four-drug sequential chemotherapy. Cancer 33 : 384-393, 1974.

4. Ng VY, Jones R, Bompadre V, Louie P, Punt S and Conrad EU III: The effect of surgery with radiation on pelvic Ewing sarcoma survival. J Surg Oncol 112: 861-865, 2015.

5. Brohl AS, Solomon DA, Chang W, Wang J, Song Y, Sindiri S, Patidar R, Hurd L, Chen L, Shern JF, et al: The genomic landscape of the Ewing Sarcoma family of tumors reveals recurrent STAG2 mutation. PLoS Genet 10: e1004475, 2014.

6. May WA, Arvand A, Thompson AD, Braun BS, Wright M and Denny CT: EWS/FLI1-induced manic fringe renders NIH 3T3 cells tumorigenic. Nat Genet 17: 495-497, 1997.

7. Delattre O,Zucman J, Plougastel B, Desmaze C, Melot T, Peter M, Kovar H, Joubert I, de Jong P, Rouleau G, et al: Gene fusion with an ETS DNA-binding domain caused by chromosome translocation in human tumours. Nature 359: 162-165, 1992.

8. Tanaka K, Iwakuma T, Harimaya K, Sato H and Iwamoto Y: EWS-Fli1 antisense oligodeoxynucleotide inhibits proliferation of human Ewing's sarcoma and primitive neuroectodermal tumor cells. J Clin Invest 99: 239-247, 1997.

9. de Alava E, Kawai A, Healey JH,Fligman I, Meyers PA, Huvos AG Gerald WL, Jhanwar SC, Argani P, Antonescu CR, et al: EWS-FLI1 fusion transcript structure is an independent determinant of prognosis in Ewing's sarcoma. J Clin Oncol 16 : $1248-1255,1998$

10. Riggi N, Knoechel B, Gillespie SM, Rheinbay E, Boulay G, Suvà $\mathrm{ML}$, Rossetti NE, Boonseng $\mathrm{WE}, \mathrm{Oksuz} \mathrm{O}, \mathrm{Cook} \mathrm{EB}$, et al: EWS-FLI1 utilizes divergent chromatin remodeling mechanisms to directly activate or repress enhancer elements in Ewing sarcoma. Cancer Cell 26: 668-681, 2014.

11. Tang SW, Bilke S, Cao L, Murai J, Sousa FG, Yamade M, Rajapakse V, Varma S, Helman LJ, Khan J, et al: SLFN11 Is a transcriptional target of EWS-FLI1 and a determinant of drug response in Ewing sarcoma. Clin Cancer Res 21: 4184-4193, 2015.

12. He T, Surdez D, Rantala JK, Haapa-Paananen S, Ban J, Kauer M, Tomazou E, Fey V, Alonso J, Kovar H, et al: High-throughput RNAi screen in Ewing sarcoma cells identifies leucine rich repeats and WD repeat domain containing 1 (LRWD1) as a regulator of EWS-FLI1 driven cell viability. Gene 596: 137-146, 2017.

13. Niedan S, Kauer M, Aryee DN, Kofler R, Schwentner R, Meier A, Pötschger U, Kontny U and Kovar H: Suppression of FOXO1 is responsible for a growth regulatory repressive transcriptional sub-signature of EWS-FLI1 in Ewing sarcoma. Oncogene 33: 3927-3938, 2014

14. Tancredi R, Zambelli A, DaPrada GA, Fregoni V, Pavesi L, Riccardi A, Burdach S, Grohar PJ and D'Incalci M: Targeting the EWS-FLI1 transcription factor in Ewing sarcoma. Cancer Chemother Pharmacol 75: 1317-1320, 2015.

15. Gupta AK, Fleckman P and Baran R: Ciclopirox nail lacquer topical solution $8 \%$ in the treatment of toenail onychomycosis. J Am Acad Dermatol 43 (4 Suppl): S70-S80, 2000.

16. Bohn M and Kraemer KT: Dermatopharmacology of ciclopirox nail lacquer topical solution $8 \%$ in the treatment of onychomycosis. J Am Acad Dermatol 43 (4 Suppl): S57-S69, 2000.
17. Clement PM, Hanauske-Abel HM, Wolff EC, Kleinman HK and Park MH: The antifungal drug ciclopirox inhibits deoxyhypusine and proline hydroxylation, endothelial cell growth and angiogenesis in vitro. Int J Cancer 100: 491-498, 2002

18. Zhou H, Shen T, Luo Y, Liu L, Chen W, Xu B, Han X, Pang J, Rivera CA and Huang S: The antitumor activity of the fungicide ciclopirox. Int J Cancer 127: 2467-2477, 2010.

19. Sen S, Hassane DC, Corbett C, Becker MW, Jordan CT and Guzman ML: Novel mTOR inhibitory activity of ciclopirox enhances parthenolide antileukemia activity. Exp Hematol 41: 799-807.e4, 2013.

20. Bilke S, Schwentner R, Yang F, Kauer M, Jug G, Walker RL, Davis S, Zhu YJ, Pineda M, Meltzer PS and Kovar H: Oncogenic ETS fusions deregulate E2F3 target genes in Ewing sarcoma and prostate cancer. Genome Res 23: 1797-1809, 2013.

21. Goss KL and Gordon DJ: Gene expression signature based screening identifies ribonucleotide reductase as a candidate therapeutic target in Ewing sarcoma. Oncotarget 7: 63003-63019, 2016.

22. Yang YH, Dudoit S, Luu P, Lin DM, Peng V, Ngai J and Speed TP: Normalization for cDNA microarray data: A robust composite method addressing single and multiple slide systematic variation. Nucleic Acids Res 30: e15, 2002.

23. Wang J, Vasaikar S, Shi Z, Greer M and Zhang B: WebGestalt 2017: A more comprehensive, powerful, flexible and interactive gene set enrichment analysis toolkit. Nucleic Acids Res: May 3 2017, DOI: 10.1093/nar/gkx356.

24. Supek F, Bošnjak M, Škunca N and Šmuc T: REVIGO summarizes and visualizes long lists of gene ontology terms. PLoS One 6: e21800, 2011.

25. Langmead B and Salzberg SL: Fast gapped-read alignment with Bowtie 2. Nat Methods 9: 357-359, 2012.

26. Feng J, Liu T, Qin B, Zhang Y and Liu XS: Identifying ChIP-seq enrichment using MACS. Nat Protoc 7: 1728-1740, 2012.

27. Yu G, Wang LG and He QY: ChIPseeker: An R/Bioconductor package for ChIP peak annotation, comparison and visualization. Bioinformatics 31: 2382-2383, 2015.

28. Thorvaldsdóttir H, Robinson JT and Mesirov JP: Integrative genomics viewer (IGV): High-performance genomics data visualization and exploration. Brief Bioinform 14: 178-192, 2013.

29. Szklarczyk D, Morris JH, Cook H, Kuhn M, Wyder S, Simonovic M, Santos A, Doncheva NT, Roth A, Bork P, et al: The STRING database in 2017: Quality-controlled protein-protein association networks, made broadly accessible. Nucleic Acids Res 45: D362-D368, 2017.

30. Shannon P, Markiel A, Ozier O, Baliga NS, Wang JT, Ramage D, Amin N, Schwikowski B and Ideker T: Cytoscape: A software environment for integrated models of biomolecular interaction networks. Genome Res 13: 2498-2504, 2003.

31. Dittmar W and Lohaus G: HOE 296, a new antimycotic compound with a broad antimicrobial spectrum. Laboratory results. Arzneimittelforschung 23: 670-674, 1973.

32. Ceschin-Roques CG, Hänel H, Pruja-Bougaret SM, Luc J, Vandermander J and Michel G: Ciclopirox nail lacquer 8\%: In vivo penetration into and through nails and in vitro effect on pig skin. Skin Pharmacol 4: 89-94, 1991.

33. Gupta AK: Ciclopirox: An overview. Int J Dermatol 40: 305-310, 2001.

34. Minden MD, Hogge DE, Weir SJ, Kasper J, Webster DA, Patton L, Jitkova Y, Hurren R, Gronda M, Goard CA, et al: Oral ciclopirox olamine displays biological activity in a phase I study in patients with advanced hematologic malignancies. Am J Hematol 89: 363-368, 2014.

35. Zhou HY, Shen T, Luo Y, Liu L, Chen W, Xu B, Han X, Pang J, Rivera CA and Huang S: The antitumor activity of the fungicide ciclopirox. Int J Cancer 10: 2467-2477, 2010.

36. Eberhard Y, McDermott SP, Wang X, Gronda M, Venugopal A, Wood TE, Hurren R, Datti A, Batey RA, Wrana J, et al: Chelation of intracellular iron with the antifungal agent ciclopirox olamine induces cell death in leukemia and myeloma cells. Blood 114: 3064-3073, 2009.

37. Grohar PJ, Segars LE, Yeung C, Pommier Y, D'Incalci M, Mendoza A and Helman LJ: Dual targeting of EWS-FLI1 activity and the associated DNA damage response with trabectedin and SN38 synergistically inhibits Ewing sarcoma cell growth. Clin Cancer Res 20: 1190-1203, 2014.

38. Grohar PJ, Kim S, Rangel Rivera GO, Sen N, Haddock S, Harlow ML, Maloney NK, Zhu J, O'Neill M, Jones TL, et al: Functional genomic screening reveals splicing of the EWS-FLI1 fusion transcript as a vulnerability in Ewing Sarcoma. Cell Rep 14: 598-610, 2016. 
39. Minas TZ, Surdez D, Javaheri T, Tanaka M, Howarth M, Kang HJ, Han J, Han ZY, Sax B, Kream BE, et al: Combined experience of six independent laboratories attempting to create an Ewing sarcoma mouse model. Oncotarget 8: 34141-34163, 2016.

40. Asano Y, Markiewicz M, Kubo M, Szalai G, Watson DK and Trojanowska M: Transcription factor Flil regulates collagen fibrillogenesis in mouse skin. Mol Cell Biol 29: 425-434, 2009.

41. Tsai YP and Wu KJ: Hypoxia-regulated target genes implicated in tumor metastasis. J Biomed Sci 19: 102, 2012.

42. Elzi DJ, Song M, Hakala K, Weintraub ST and Shiio Y: Proteomic analysis of the EWS-Fli-1 interactome reveals the role of the Lysosome in EWS-Fli-1 turnover. J Proteome Res 13: 3783-3791, 2014.

43. Cutroneo KR, White SL, Phan SH and Ehrlich HP: Therapies for bleomycin induced lung fibrosis through regulation of TGF-betal induced collagen gene expression. J Cell Physiol 211: 585-589, 2007.

44. Chen SJ, Yuan W, Mori Y, Levenson A, Trojanowska M and Varga J: Stimulation of type I collagen transcription in human skin fibroblasts by TGF-beta: Involvement of Smad 3. J Invest Dermatol 112: 49-57, 1999.

45. Verrecchia F, Chu ML and Mauviel A: Identification of novel TGF- $\beta /$ Smad gene targets in dermal fibroblasts using a combined cDNA microarray/promoter transactivation approach. J Biol Chem 276: 17058-17062, 2001.

46. Hahm KB: Repression of the gene encoding the TGF-beta type II receptor is a major target of the EWS-FLI1 oncoprotein. Nat Genet 23: 481, 1999.

47. Semenza GL: Hypoxia-inducible factor 1: Oxygen homeostasis and disease pathophysiology. Trends Mol Med 7: 345-350, 2001
48. Liu LZ, Hu XW, Xia C, He J, Zhou Q, Shi X, Fang J and Jiang BH: Reactive oxygen species regulate epidermal growth factor-induced vascular endothelial growth factor and hypoxia-inducible factor-1 alpha expression through activation of AKT and P70S6K1 in human ovarian cancer cells. Free Radic Biol Med 41: 1521-1533, 2006.

49. Simon MC and Keith B: The role of oxygen availability in embryonic development and stem cell function. Nat Rev Mol Cell Biol 9: 285-296, 2008.

50. Low-Marchelli JM, Ardi VC, Vizcarra EA, van Rooijen N, Quigley JP and Yang J: Twist1 induces CCL2 and recruits macrophages to promote angiogenesis. Cancer Res 73: 662-671, 2013.

51. Gort EH, van Haaften G, Verlaan I, Groot AJ, Plasterk RH, Shvarts A, Suijkerbuijk KP, van Laar T, van der Wall E, Raman V, et al: The TWIST1 oncogene is a direct target of hypoxia-inducible factor-2alpha. Oncogene 27: 1501-1510, 2008

52. Dehne N and Brüne B: Sensors, transmitters, and targets in mitochondrial oxygen shortage-a hypoxia-inducible factor relay story. Antioxidants Redox Signal 20: 339-352, 2014.

53. Zhdanov AV, Waters AH, Golubeva AV and Papkovsky DB: Differential contribution of key metabolic substrates and cellular oxygen in HIF signalling. Exp Cell Res 330: 13-28, 2015.

54. Somers GR, Zielenska M, Abdullah S, Sherman C, Chan S and Thorner PS: Expression of MYCN in pediatric synovial sarcoma. Modern Pathol 20: 734-741, 2007. International (CC BY-NC-ND 4.0) License. 